Das neue Reagens (2) ergibt mit Elektrophilen die in Tabelle 1 zusammengestellten Produkte ( 3 ) und ( $4 a$ ). Die mit Carbonylverbindungen erhaltenen Diole $(3 d)-(3 g)$ cyclisieren mit Salzsäure/Ether ${ }^{[5]}$ zu den Dihydroisobenzofuranen $(4 b)$ (4e) (Tabelle 1, unten).<smiles>OCc1ccccc1F</smiles>

(3)<smiles>[R]C1([R])OCc2ccccc21</smiles>

(4)<smiles>[R]C([R])(O)c1ccccc1C(O)CCCCC</smiles>

(5)
[2] Übersicht bis Anfang 1976: H. P. Abicht, K. Issleib, Z. Chem. 17, 1 (1977).

[3] D. Seebach, K. H. Geiss, Angew. Chem. 86, 202 (1974); Angew. Chem. Int. Ed. Engl. 13, 202 (1974); K. H. Geiss, D. Seebach, B. Seuring, Chem. Ber. 110,1833 (1977).

[4] a) meta-Methoxyderivat: $M$ Uemura $S$ Tokuyama, $T$. Sakan, Chem. Lett. 1975,1195 ; b) ortho-Metallierung von $\alpha$-Hydroxyphenethylaminen: D. W. Slocum, W. Achermann, J. Chem. Soc. Chem. Commun. 1974, 968; c) zur andersartig verlaufenden Reaktion von Allylalkoholat mit Organolithium- oder -magnesiumverbindungen siehe: H. Felkin, G. Swierczewski, A. Tambuté, Tetrahedron Lett. 1969, 707; J. K. Crandall, A. C. Clark, J. Org. Chem. 37, 4236 (1972); zit. Lit.

[5] H. C. Brown, K. LeRoi Nelson, J. Am. Chem. Soc. 75, 24 (1953).

Tabelle 1. Produkte vom Typ (3), (4) und (5) aus Benzylalkoholen und Alkylhalogeniden oder Carbonylverbindungen. Die Ausbeuten an $(3 a)-(3 g)$ und $(5 a)-(5 d)$ beziehen sich auf das Elektrophil. Als Siedepunkte sind Luftbadtemperaturen bei Kugelrohrdestillation (Büchi-GKR-50) angegeben.

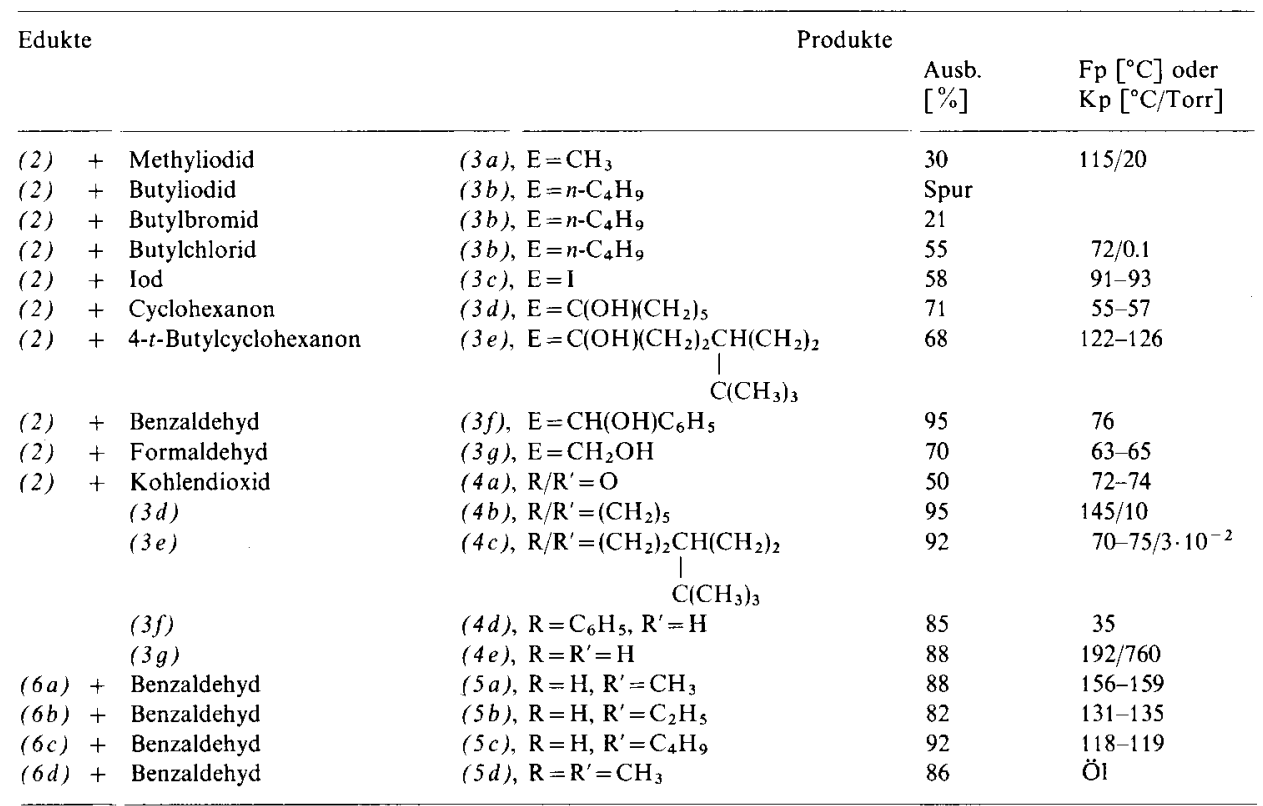

Die physikalischen Daten der bereits bekannten Verbindungen $[(3 a),(3 c),(3 f),(3 g),(4 a),(4 d),(4 e)]$ stimmen mit Literaturangaben überein. Elementaranalysen sowie IR- und NMR-Spektren bestätigen die angegebenen Strukturen. Eine Doppelmetallierung von $\alpha, \alpha$-Dideuteriobenzylalkohol beweist, daß sich (2) nicht über (1), $\mathrm{X}=\mathrm{O}$, bildet. Andere $\alpha$-Phenylalkanole reagieren wie Benzylalkohol. So erhielten wir über die ortho-lithiierten Alkoholate $(6 a)-(6 d)$ (Tabelle 1) von 1-Phenylethanol, -1-propanol, -1-pentanol bzw. 2-Phenyl-2-propanol mit Benzaldehyd die Benzhydrole $(5 a),(5 b)$, (5c) (Diastereomerengemische) bzw. (5d).

\section{Allgemeine Arbeitsvorschrift}

Zur heftig gerührten Mischung aus $10 \mathrm{mmol}$ Benzylalkohol, $20 \mathrm{ml}$ Petrolether $\left(\mathrm{Kp}=30-40^{\circ} \mathrm{C}\right)$ und $20 \mathrm{mmol}$ Tetramethylethylendiamin (TMEDA) gibt man bei Raumtemperatur unter Schutzgas $20 \mathrm{mmol} n$-Butyllithiumlösung (ca. $1.5 \mathrm{M}$ in Hexan), die erste Hälfte in $10 \mathrm{~min}$, die zweite in einem Schuß. Nach $11 \mathrm{~h}$ Erhitzen unter Rückfluß wird auf $-78^{\circ} \mathrm{C}$ abgekühlt, und es werden $7 \mathrm{mmol}$ Elektrophil zugesetzt. Man läßt auf Raumtemperatur erwärmen, gießt in verdünnte Schwefelsäure und arbeitet wie üblich mit Ether auf.

[1] H. Gilman, A. L. Jacoby, J. Org. Chem. 3, 108 (1938); H. Gilman, G. E. Brown, F. J. Webb, S. M. Spatz, J. Am. Chem. Soc. 62, 977 (1940); G. Wittig, U. Pockels, H. Dröge, Ber. Dtsch. Chem. Ges. 71, 1903 (1938); G. Wittig, Angew. Chem. 53, $241(1940)$

\section{Photochemische reduktive trans-Eliminierung bei trans- Diazidotetracyanoplatinat(IV ${ }^{[* *]}$}

Von Arnd Vogler, Alfred Kern und Jürgen Hüttermann ${ }^{[*]}$

Oxidative Additionen und reduktive Eliminierungen sind wichtige Reaktionen von Übergangsmetallkomplexen und von besonderer Bedeutung in der Chemie metallorganischer Verbindungen. Reduktive cis-Eliminierungen wurden auch als lichtinduzierte Prozesse beobachtet ${ }^{[1]}$. So führt die Bestrahlung von $\left[\mathrm{IrClH}_{2}\left(\mathrm{PPh}_{3}\right)_{3}\right]$ in einem einzigen Schritt zur cis-Eliminierung von $\mathrm{H}_{2}$ unter Bildung von $\left[\mathrm{IrCl}\left(\mathrm{PPh}_{3}\right)_{3}\right]$; die $\mathrm{H}_{2}-\mathrm{Ab}$ spaltung verläuft dabei nicht über H-Atome ${ }^{[1 b]}$. Photochemische reduktive trans-Eliminierungen waren bisher unbekannt. Wir konnten am Beispiel von $\left[\mathrm{Pt}^{\mathrm{IV}}(\mathrm{CN})_{4}\left(\mathrm{~N}_{3}\right)_{2}\right]^{2-}$, das durch Belichtung vollständig in $\left[\mathrm{Pt}^{\mathrm{II}}(\mathrm{CN})_{4}\right]^{2-}$ umgewandelt wird, nun erstmals eine solche Reaktion nachweisen.

Besonders interessierte uns, ob diese Zweielektronenreduktion von $\mathrm{Pt}^{\mathrm{IV}}$ über eine $\mathrm{Pt}^{\mathrm{III}}$-Z $\mathrm{Z}$ wischenstufe abläuft, da allgemein angenommen wird, daß sowohl thermische ${ }^{[2 a]}$ als auch photochemische ${ }^{[2 \mathrm{~b}]}$ Umwandlungen von $\mathrm{Pt}^{\mathrm{IV}}-\mathrm{zu} \mathrm{Pt}^{\mathrm{II}}-\mathrm{Komplexen}$ aus zwei aufeinanderfolgenden Einelektronenreduktionen be-

$\left[{ }^{*}\right]$ Prof. Dr. A. Vogler, Dipl.-Chem. A. Kern

Institut für Chemie der Universität

Universitätsstraße 31, D-8400 Regensburg 2

Prof. Dr. J. Hüttermann

Institut für Biophysik und Physikalische Biochemie der Universität Regensburg

$\left[{ }^{* *}\right]$ Diese Arbeit wurde von der Deutschen Forschungsgemeinschaft und vom Fonds der Chemischen Industrie unterstützt. 
stehen. Ohne $\mathrm{Pt}^{\mathrm{III}}$-Zwischenstufe gibt es zwei mechanistische Möglichkeiten: Während bei cis-Eliminierung die Abspaltung der beiden Liganden offensichtlich nicht mit einer Bildung von Ligandradikalen im primären Reaktionsschritt einhergeht ${ }^{[1 \mathrm{~b}]}$, könnte dies bei trans-Eliminierung aus sterischen Gründen der Fall sein.

Unsere Untersuchungen haben ergeben, daß im photochemischen Primärschritt der trans-Eliminierung an trans$\left[\mathrm{Pt}(\mathrm{CN})_{4}\left(\mathrm{~N}_{3}\right)_{2}\right]^{2-}$ durch gleichzeitige Zweielektronenreduktion unter Umgehung einer $\mathrm{Pt}^{\mathrm{III}}$-Zwischenstufe zwei $\mathrm{N}_{3}$-Radikale gebildet werden:

\section{$\left[\mathrm{Pt}^{\mathrm{IV}}(\mathrm{CN})_{4}\left(\mathrm{~N}_{3}\right)_{2}\right]^{2-} \rightarrow\left[\mathrm{Pt}^{\mathrm{II}}(\mathrm{CN})_{4}\right]^{2-}+2 \mathrm{~N}_{3}^{*}$}

Die Verbindung $\mathrm{K}_{2}\left[\mathrm{Pt}(\mathrm{CN})_{4}\left(\mathrm{~N}_{3}\right)_{2}\right]$ wurde aus $\mathrm{K}_{2}\left[\mathrm{Pt}(\mathrm{CN})_{4} \mathrm{Br}_{2}\right]^{[3]}$ und einem großen Überschuß von $\mathrm{KN}_{3}$ in Wasser (Reaktionszeit $2 \mathrm{~d}$ ) nach Umkristallisation analysenrein erhalten. Für die trans-Struktur $\left(\mathrm{D}_{4 \mathrm{~h}}\right)$ des komplexen Anions spricht eine IR-Bande ${ }^{[3]}\left(2181 \mathrm{~cm}^{-1}\right)$ im Bereich der $\mathrm{CN}$-Valenzschwingungen. Das Elektronenabsorptionsspektrum weist im langwelligen Teil nur ein intensives Maximum $(\lambda=302 \mathrm{~nm}, \varepsilon=18300)$ auf, das einem $\left(\mathrm{N}_{3} \rightarrow \mathrm{Pt}\right) \mathrm{CT}$-Übergang zugeordnet wird.

Bestrahlung der CT-Bande des in Wasser gelösten Salzes führte zur raschen Entwicklung von Stickstoff nach

$\left[\mathrm{Pt}(\mathrm{CN})_{4}\left(\mathrm{~N}_{3}\right)_{2}\right]^{2-} \rightarrow\left[\mathrm{Pt}(\mathrm{CN})_{4}\right]^{2-}+3 \mathrm{~N}_{2}$

Die spektralen Veränderungen während der Photolyse (Abb. 1) zeigen, daß keine anderen Produkte entstehen, da das Spektrum nach längerer Belichtungsdauer identisch mit dem von $\left[\mathrm{Pt}(\mathrm{CN})_{4}\right]^{2-[4]}$ war; auch freies Azid war nicht nachweisbar ${ }^{[5]}$. Bei vollständiger Lichtabsorption durch den Ausgangskomplex folgte die photochemische Bildung von $\left[\mathrm{Pt}(\mathrm{CN})_{4}\right]^{2-}$ einer Reaktion nullter Ordnung. Die Quantenausbeuten für $\left[\mathrm{Pt}(\mathrm{CN})_{4}\right]^{2-}$ in Wasser betrugen bei den Anregungswellenlängen 300,333 und $366 \mathrm{~nm} \Phi=0.34 \pm 0.02$, in Acetonitril oder Ethanol stiegen sie auf $\Phi=0.58$ bzw. $0.61 \pm 0.02$ an. Im Unterschied zur wäßrigen Lösung wurde in Ethanol neben $\mathrm{N}_{2}$ auch $\mathrm{N}_{3}^{-}$als Photolyseprodukt gefunden.

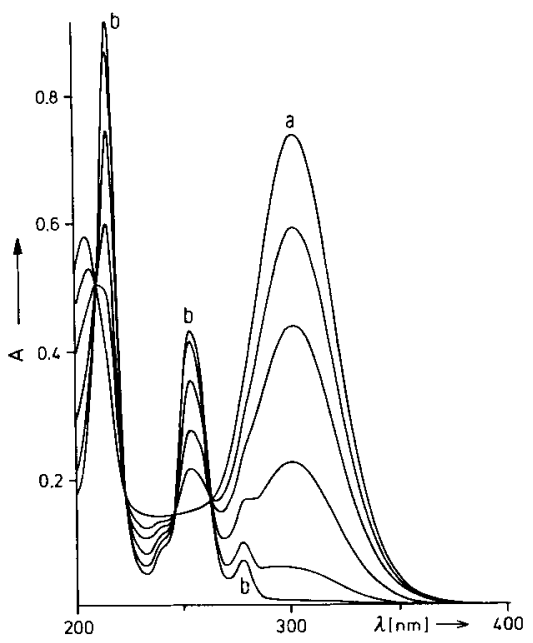

Abb. 1. Veränderung des Absorptionsspektrums während der photochemischen Umwandlung von trans- $\left[\mathrm{Pt}(\mathrm{CN})_{4}\left(\mathrm{~N}_{3}\right)_{2}\right]^{2-}$ (a) in PtiC $\mathrm{N}_{4}$ (b) in wäßri-

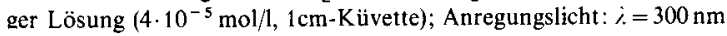

Zur Identifizierung der Primärprodukte wurde die Photolyse von $\left[\mathrm{Pt}(\mathrm{CN})_{4}\left(\mathrm{~N}_{3}\right)_{2}\right]^{2-}$ in Ethanolgläsern bei $77 \mathrm{~K}$ ESR-spektroskopisch untersucht. Die beobachteten Signale konnten N-Atomen ${ }^{[6]}$ und $\mathrm{CH}_{3} \mathrm{CHOH}-\mathrm{Radikalen}{ }^{[7]}$ zugeordnet werden. $\mathrm{Pt}^{\mathrm{III}}$-Signale ${ }^{[8]}$ traten selbst bei $20 \mathrm{~K}$ nicht auf.
$\mathrm{N}_{3}$-Radikale sind extrem instabil. Sie zerfallen noch bei tiefen Temperaturen sehr schnell in molekularen Stickstoff und N-Atome ${ }^{[6 b, c]}$, die ESR-spektroskopisch nachgewiesen wurden. Bei Raumtemperatur wird in wäßriger Lösung neben $\left[\mathrm{Pt}(\mathrm{CN})_{4}\right]^{2-}$ schließlich nur $\mathrm{N}_{2}$ als stabiles Endprodukt gebildet. In Anwesenheit geeigneter Reduktionsmittel können $\mathrm{N}_{3^{-}}$ Radikale zu Azid reduziert werden ${ }^{[9]}$. Die Entstehung der $\mathrm{CH}_{3} \mathrm{CHOH}$-Radikale, die ebenfalls bei tiefen Temperaturen identifiziert wurden, und von freiem Azid bei der Raumtemperaturphotolyse von $\left[\mathrm{Pt}(\mathrm{CN})_{4}\left(\mathrm{~N}_{3}\right)_{2}\right]^{2-}$ in Ethanol läßt sich nur durch eine Konkurrenzreaktion der $\mathrm{N}_{3}$-Radikale erklären: Neben dem Zerfall können die $\mathrm{N}_{3}$-Radikale offenbar vom Lösungsmittel $\mathrm{CH}_{3} \mathrm{CH}_{2} \mathrm{OH}$ reduziert werden.

$$
\begin{aligned}
& \text { Eingegangen am 17. Dezember 1976, } \\
& \text { in geänderter Fassung am 13. April } 1978 \text { [Z 987] }
\end{aligned}
$$

[1] a) G. L. Geoffroy, G. S. Hammond, H. B. Gray, J. Am. Chem. Soc. 97, 3933 (1975); b) G. L. Geoffroy, R. Pierantozzi, ibid. 98, 8054 (1976); c) M.S. Wrighton, Top. Curr. Chem. 65, 37 (1976).

[2] a) F. Basolo, R. G. Pearson: Mechanisms of Inorganic Reactions, 2nd Ed. Wiley, New York 1967 ; b) P. C. Ford, R. E. Hintze, J. D. Petersen in A. W. Adamson, P. D. Fleischauer: Concepts of Inorganic Photochemistry. Wiley-Interscience, New York 1975.

[3] L. H. Jones, J. M. Smith, Inorg. Chem. 4, 1677 (1965).

[4] S. B. Piepho, P. N. Schatz, A. J. McCaffery, J. Am. Chem. Soc. 91, 5994 (1969).

[5] E. K. Dukes, R. M. Wallace, Anal. Chem. 33, 242 (1961).

[6] a) A. Begum, M.C.R. Symons, J. Chem. Soc. A 1971, 2062; b) I. S. Ginns, M. C. R. Symons, J. Chem. Soc. Faraday Trans. II 68, 631 (1972); c) T. A. Claxton, R. E. Overill, M. C. R. Symons, Mol. Phys. 26, 75 (1973).

[7] P. J. Sullivan, W. S. Koski, J. Am. Chem. Soc. 86, 159 (1964).

[8] T. Krigas, M. T. Rogers, J. Chem. Phys. 55, 3035 (1971); C. Amano, S. Fujiwara, Bull. Chem. Soc. Jpn. 50, 1437 (1977).

[9] J. F. Endicott, M. Z. Hoffmann, L. S. Beres, J. Phys. Chem. 74, 1021 (1970).

\section{Hochdruckcarbonylierung metallkoordinierter Carbene und Hydrogenolyse der Keten-Komplexe ${ }^{[1]}$}

Von Wolfgang A. Herrmann und Johann Plank ${ }^{[*]}$

Die Reaktivität von Übergangsmetall-Carben-Komplexen gegenüber Kohlenmonoxid ${ }^{[2 a]}$ und Wasserstoff ${ }^{2 b]}$ ist insbesondere im Zusammenhang mit der neuerdings wieder aktuellen Fischer-Tropsch-Synthese von Interesse ${ }^{[3]}$. Während mit beiden Substraten bisher nur die Ablösung des Carben-Liganden vom Metall beobachtet worden is ${ }^{[2]}$, gelang uns jetzt erstmals die Carbonylierung metallkoordinierter Carbene zu den entsprechenden Keten-Komplexen sowie auch deren hydrogenolytischer Abbau zu Aldehyden und Alkoholen.

Aus den Diphenylcarben-Mangan-Komplexen $(1)^{[4]}$ entstehen unter den Bedingungen einer Hochdruckcarbonylierung (CO-Addition mit 30-45\% Umsatz) die Diphenylketen-Komplexe (2). Ihre physikalischen und spektroskopischen Daten stimmen mit denen der aus Diphenylketen und den Tetrahy-

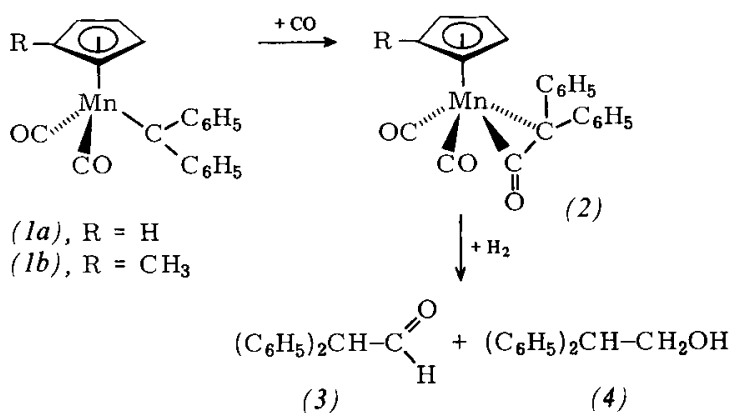

[*] Dr. W. A. Herrmann, J. Plank

Chemisches Institut der Universität

Universitätsstraße 31, D-8400 Regensburg 1 